\title{
Freaks or Foods: What motivates alternative nutrition and lifestyles?
}

\section{Abstract}

Globally, the adverse effects of increased animal-protein consumption on the environment, the society, and the economy are a cause of growing societal concern. As a result, issues of food system sustainability have received considerable attention within the national and international community. For the European community, changes in food lifestyles of consumers, in particular a reduced share of animalbased foods within the diet, are of great importance for the establishment of sustainable food supply structures. Meanwhile, a worldwide trend for alternative nutrition and lifestyles is obvious, which is reflected by a growing number of plant-based nutrition styles and craftfoods. Often, alternative nutrition styles are ethically or health motivated. Nonetheless, changes in regard to nutrition are often not consistent. Up to now, research mainly focused on the quantitative and qualitative aspects of food lifestyle changes. However, extreme, alternative nutrition lifestyles seem to be more disciplined with regard to nutrition transformation. Nonetheless, especially extreme food consumption patterns have not been assessed with regard to a long-lasting sustainable transformation potential. However, we propose that a comprehensive framework of these issues must contain information on the specific motivational themes that lead people to make substantial changes in their diets. Hence, the objective of the proposed study is to assess the transforming potential by in-depth interviews with extreme alternative nutrition styles. We aim in gathering knowledge on the content of the motivational dynamics influencing consumers in adapting alternative food lifestyles like raw-foodism, Paleolithic diet, or Anthroposophic diet. The theoretical framework applied is grounded in self-determination theory, a meta-theoretical construct used for explaining motivational change dynamics in humans. The research questions that will be addressed are: "What motivates people to adapt extreme alternative dietary styles?", "How do identity and individual worldview influence the transition process?" and "Which strategies were applied to successfully implement new dietary habits into daily routine? In-depth interviews, following an experience interview will be conducted with 18 participants who had practiced an alternative food lifestyle for a long-term period. The transcripts of these interviews will be structured using content analysis. Since data collection will take place in May 2019 results cannot be described by deadline for the call of abstracts. Results and conclusion that will be addressed in the FENS Conference will include the motivational drivers for long-lasting transformation potential and discuss the applicability for society and behavior change communication.

\section{Conflict of Interest}

There is no conflict of interest 